\title{
ARRB1 enhances the chemosensitivity of lung cancer through the mediation of DNA damage response
}

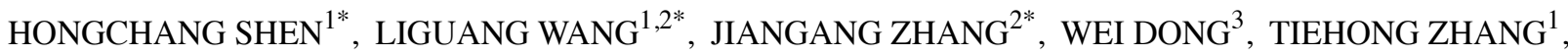 \\ YANG NI ${ }^{1}, \mathrm{HONGXIN} \mathrm{CAO}^{2}, \mathrm{KAI} \mathrm{WANG}^{2}, \mathrm{YUN} \mathrm{LI}^{3}, \mathrm{YIBING} \mathrm{WANG}^{4}$ and JIAJUN DU ${ }^{2,3}$ \\ ${ }^{1}$ Department of Oncology, ${ }^{2}$ Institute of Oncology, and Departments of ${ }^{3}$ Thoracic Surgery and ${ }^{4}$ Surgery, \\ Shandong Provincial Hospital Affiliated to Shandong University, Shandong University, \\ Jinan, Shandong 250021, P.R. China
}

Received September 18, 2016; Accepted October 21, 2016

DOI: $10.3892 /$ or.2016.5337

\begin{abstract}
ARRB1 (also known as $\beta$-arrestin-1) serves as a multifunctional adaptor contributing to the regulation of signaling pathways. ARRB1 may be involved in DNA damage accumulation; however the underlying mechanism involved is unclear. In the present study, non-small cell lung cancer (NSCLC) cell lines (H520 and SK-MES-1) were transfected with ARRB1 plasmids or small interfering ribonucleic acid (siRNA) and received treatment with DNA-damaging agents (cisplatin and etoposide). A mouse xenograft model was used to assess the impact of ARRB1 on the efficacy of cisplatin in vivo. A total of 30 surgically resected NSCLC patients were recruited for the present study and qRT-PCR was performed to determine the mRNA levels in cancer tissues compared with para-carcinoma tissues. Our data showed that DNA damage was abrogated in the ARRB1-knockdown cells and enhanced in the ARRB1-overexpressing cells. ATR and Chk1 were more activated in the ARRB1-overexpressing cells compared to the ARRB1-knockdown cells, followed by increased H2AX phosphorylation. DNA damage and apoptosis were increased in the ARRB1-overexpressing cells treated with cisplatin. These data provided strong evidence that ARRB1 contributes to the response of NSCLC to DNA-damaging agents and is essential for DNA damage response (DDR). ARRB1 may enhance the efficacy of DNA-damaging agents in NSCLC.
\end{abstract}

Correspondence to: Dr Jiajun Du, Department of Thoracic Surgery, Shandong Provincial Hospital Affiliated to Shandong University, Shandong University, 324 Jingwu Road, Jinan, Shandong 250021, P.R. China

E-mail: sdudujiajun@126.com

Dr Yibing Wang, Department of Surgery, Shandong Provincial Hospital Affiliated to Shandong University, Shandong University, 324 Jingwu Road, Jinan, Shandong 250021, P.R. China

E-mail:wyb0616@163.com

${ }^{*}$ Co-first authorship

Key words: $\beta$-arrestin-1, chemosensitivity, DNA damage response, lung cancer

\section{Introduction}

Lung cancer is one of the most common types of malignancies. It is not only the leading cause of cancer-related deaths, but also the most frequently diagnosed cancer worldwide (1). Yearly, it accounts for more than 1.8 million newly diagnosed cases (13\% of total) and 1.6 million cancer-related deaths (19.4\% of total) (2). Approximately $90 \%$ of all lung cancers are NSCLC, with $25-30 \%$ of the NSCLC cases being squamous cell cancer (SCC) (3). SCC is the second most common histologic subtype of NSCLC (4). Compared with other histologic types, SCC exhibits distinct epidemiological, clinicopathological and molecular characteristics. In particular, SCC lesions are usually located centrally in segmental, lobar or main bronchi, may show central cavitation and are associated with increased rates of hemorrhage compared with other lung cancer types (5). While outcomes have improved with adenocarcinoma, treatment options and outcomes are still limited in patients with advanced-stage SCC (4). To date, no agents have been specifically approved for the treatment of SCC (3).

Surgery, chemotherapy and radiotherapy are major treatment methods for lung cancer patients (6). Therein, surgery is considered as the most effective curable method, particularly for early-stage patients. However, $\sim 70 \%$ of lung cancer patients suffer from local advanced or metastatic disease at the time of diagnosis and are unsuitable to receive surgical treatment (7). Thus, receiving chemotherapy is unavoidable for these patients and the efficacy of chemotherapy is still a matter of concern. The genome DNA is constantly exposed to various genotoxic insults. The DNA damage response (DDR) is a complex signal transduction pathway that leads to multiple outcomes including cell cycle arrest, DNA repair, senescence and apoptosis (8-10). DNA-damaging agents induce various types of DNA damage including modification of bases, intra-strand crosslinks, inter-strand crosslinks, single-strand breaks (SSBs) and doublestrand breaks (DSBs). Each type of DNA damage is recognized and processed by proteins involved in the DDR. The response to DNA DSBs and collapsed replication forks is particularly crucial as these types of damage are difficult to repair. To respond to DNA damage, ataxia telangiectasia mutated (ATM) or ataxia telangiectasia and Rad3-related (ATR) phosphorylates and activates checkpoint kinase $1 / 2(\mathrm{Chk} 1 / 2)$, and then causes 
cell cycle arrest and DNA repair, otherwise it may lead to cell apoptosis when DNA damage is too severe.

Arrestins were initially discovered to interact with G proteincoupled receptors (GPCRs) to mediate their internalization and desensitization $(11,12)$. In recent years, more and more novel functions of ARRB1 have been discovered. Arrestins, including ARRB1, always serve as adaptors, scaffolds and/or signal transducers, and they connect the activated receptors with diverse signaling pathways within the cell. ARRB1 plays a vital role in scaffolding and modulating varieties of intracellular signaling networks such as NF- $\kappa \mathrm{B}$ and Mdm2/p53 (13). In this diversity of cell signaling pathways, ARRB1 may have different effects on the cellular process. Our former study showed that loss of ARRB1 expression was associated with poor survival of NSCLC particularly in SCC (14). Recently, it was reported that ARRB1 may be involved in DNA damage, which is a common target of chemotherapy and radiotherapy $(15,16)$, however, whether DDR is involved in this process remains unclear. We aimed to study the role of ARRB1 in DDR induced by DNA-damaging agents, and to explore the underlying mechanism of regulation within it.

\section{Materials and methods}

Ethical approval. Written informed consent was obtained from all the subjects involved and the present study was approved by the Shandong Provincial Hospital Ethics Committee. All methods used in the present study were carried out in accordance with the approved guidelines.

Patients. Fresh frozen tissue samples from 30 patients with primary lung squamous cancer who had undergone complete surgical resection were obtained in the present study (Table I). Patients and relevant data were acquired from the Bio-Bank of Shandong Provincial Hospital from January 2011 to December 2012.

The Ethics Community of Shandong Provincial Hospital affiliated to Shandong University permitted the undertaking of the present study and the informed consent from patients for the use of clinical data was obtained before surgery.

For the histology and tumor-node-metastasis (TNM) stage, the classification criteria for lung tumors from the World Health Organization and International Association for the Study of Lung Cancer (WHO/IASLC) was applied. For the follow-up, patients were evaluated every 3 months by thorax CT and abdomen ultrasonography for the first 2 years after surgery along with adjuvant treatment, and annually thereafter according to schedule.

Cell culture and transfection. Two human lung squamous cancer cell lines (H520 and SK-MES-1) were used in the present study. Both of them were obtained from the American Type Culture Collection (ATCC; Manassas, VA, USA). Cells were grown in monolayer cultures in RPMI-1640 medium (HyClone, Logan, UT, USA) containing 10\% fetal bovine serum (Gibco, Carlsbad, CA, USA) in a humidified $37^{\circ} \mathrm{C}$ atmosphere of $5 \% \mathrm{CO}_{2}$. For both plasmids (pcDNA and ARRB1) and siRNA transfection, all cells were seeded into 6 -well cell culture plates (diameter of each well was $\sim 35 \mathrm{~mm}$; Corning, Corning, NY, USA). When the cell density reached $\sim 50-70 \%$ confluence the next day, Lipofectamine 2000 trans-
Table I. Characteristics of the patients with SCC-NSCLC in the present study.

\begin{tabular}{|c|c|c|}
\hline Variables & $\mathrm{n}$ & $\begin{array}{c}\text { Percentage } \\
\quad(\%)\end{array}$ \\
\hline All cases & 30 & 100.00 \\
\hline \multicolumn{3}{|c|}{ Age at diagnosis (years) } \\
\hline$\geq 60$ & 21 & 70.00 \\
\hline$<60$ & 9 & 30.00 \\
\hline \multicolumn{3}{|l|}{ Gender } \\
\hline Female & 5 & 16.67 \\
\hline Male & 25 & 83.33 \\
\hline \multicolumn{3}{|c|}{ Smoking index } \\
\hline$\geq 400$ & 23 & 76.67 \\
\hline$<400$ & 7 & 23.33 \\
\hline \multicolumn{3}{|c|}{ Tumor differentiation } \\
\hline Well & 0 & 0.00 \\
\hline Moderate & 21 & 70.00 \\
\hline Poor & 9 & 30.00 \\
\hline \multicolumn{3}{|c|}{ Pathological stage } \\
\hline $\mathrm{I}+\mathrm{II}$ & 5 & 16.67 \\
\hline III+IV & 25 & 83.33 \\
\hline \multicolumn{3}{|c|}{ Lymph node status } \\
\hline Negative & 18 & 60.00 \\
\hline Positive & 12 & 40.00 \\
\hline
\end{tabular}

SCC, squamous cell cancer; NSCLC, non-small cell lung cancer.

fection reagent (Invitrogen, Carlsbad, CA, USA) was applied according to the manufacturer's instructions.

MTT and colony formation assays. The 3-(4,5-dimethylthiazol-2-yl)-2,5-diphenyltetrazolium bromide (MTT) assay was used to assess the cytotoxicity of DNA-damaging agents. Cells were seeded into 96-well culture plates with a density of $3 \times 10^{3}$ and incubated for $24 \mathrm{~h}$ under the conditions described above for standard cell culture maintenance. Then, the cells received cisplatin or etoposide treatment at different doses. After a 48-h treatment, $10 \mu \mathrm{l}$ MTT reagent was added into each well and incubated for another $4 \mathrm{~h}$. Finally, $100 \mu \mathrm{l}$ dimethyl sulfoxide (DMSO) was added into each of the same wells. Then, the optical density value of each well was determined using a Bio-Rad model 680 microplate reader at a wavelength of $490 \mathrm{~nm}$.

As for the colony formation assay, cells were plated into 12 -well culture plates with 1,000 cells in each well. On the following day, the cells received drugs $(1.5 \mu \mathrm{M}$ cisplatin or etoposide) and were then incubated for 14 days. Subsequently, the plates were stained with crystal violet, and a colony count was conducted using ImageJ software.

Western blotting and co-immunoprecipitation (Co-IP). Western blotting was performed to detect the specific protein levels (total or phosphorylated). Cells were lysed in RIPA buffer supplemented with PhosSTOP (Roche, Mannheim, Germany) 
on ice. After BCA protein quantification, loading buffer was added into the mixture. Then, a standard western blotting procedure was performed. Nitrocellulose filter membranes with proteins were covered with primary antibodies overnight. After washing the membrane 3 times with Tris-buffered saline and Tween-20 (TBST), horseradish peroxidase (HRP)-conjugated anti-mouse, anti-goat or anti-rabbit antibodies were applied according to the primary antibodies on the following day. After washing the membranes 3 times with TBST, the protein levels were detected using enhanced chemiluminescence (ECL) detection reagent (Advansta Corp., Menlo Park, CA, USA).

For Co-IP, cells after treatment were lysed by RIPA solution for Co-IP on ice for $30 \mathrm{~min}$. Then, BCA protein quantification was conducted. Each adjusted buffer was added to $5 \mu \mathrm{l}$ anti-ARRB1 antibody (Santa Cruz Biotechnology, Santa Cruz, CA, USA) and protein A and G Sepharose beads (Invitrogen) overnight. The beads were washed 3 times with immunecomplex buffer and resolved by SDS-PAGE. Then $1 \mathrm{X}$ sample buffer was added to the resolved precipitates and the mixture was boiled for $10 \mathrm{~min}$. Subsequently, western blotting was performed.

Antibodies used in the western blotting are listed as follows. Anti-GAPDH antibody was purchased from Santa Cruz Biotechnology. Anti-caspase3, -PARP, -ARRB1 and anti-phosphorylated-ATR (Ser428), -ATM (Ser1981), -Chk1 (Ser345), -Chk2 (Thr68), -BRCA1 (Ser1524), -H2AX (Ser139) and $-\mathrm{NF}-\kappa \mathrm{B}$ p65 (Ser536) antibodies were all purchased from Cell Signaling Technology (Danvers, MA, USA). Anticancer drugs (cisplatin and etoposide) were purchased from Sigma-Aldrich (St. Louis, MO, USA).

Flow cytometry. PE-Annexin V/7-amino-actinomycin D (7-AAD) staining assay was used to assess apoptosis. Annexin $\mathrm{V}$ is a phospholipid-binding protein with a high affinity for phosphatidylserine (PS). In normal live cells, PS is located on the cytoplasmic surface of the cell membrane. When cells undergo apoptosis, PS is translocated from the inner to the outer leaflet of the plasma membrane, and becomes available for Annexin V binding (17). 7-AAD is generally cell-impermeable for live cells, and undergoes a spectral shift upon association with DNA. Thus, it has been used to label necrotic or late apoptotic/dead cells with damaged cell membranes (18). Therefore, combination of PE-Annexin V and 7-AAD stains can be exploited to distinguish cells undergoing necrosis and apoptosis to cell death (19). Apoptosis was detected in the present study, using flow cytometry (FCM). A PE Annexin V apoptosis detection kit (559763) and a LSRFortessa (both from BD Pharmingen, Franklin Lakes, NJ, USA) were used for FCM. No adjustments were made to the instrument configuration and settings.

Comet assay. The comet assay (also called single-cell gel electrophoresis) is a sensitive and rapid method for DNA strand-break detection in eukaryotic cells (20). We performed the assay according to the instructions (Cell Biolabs Inc., San Diego, CA, USA) to evaluate the DNA damage induced by chemotherapy. Cells were digested by trypsin and washed twice with cold phosphate-buffered saline (PBS). Then, OxiSelect Comet Slides were covered with a mixture of comet agarose and the cells. After lysis, the slides underwent electrophoresis

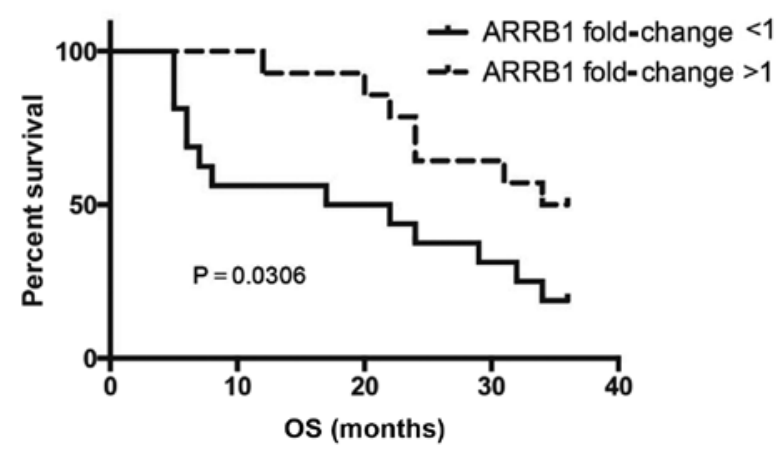

Figure 1. Kaplan-Meier survival curve for the overall survival (OS) of 30 SCC patients. Those patients with low levels of ARRB1 showed significantly poorer $\mathrm{OS} ; \mathrm{P}=0.0306$

for $30 \mathrm{~min}$ under $1 \mathrm{~V} / \mathrm{cm}$ at $4^{\circ} \mathrm{C}$. Cells were stained with Vista Green DNA dye and visualized using epifluorescence microscopy. The percentage of DNA migrated in the tail (\% of tail DNA), the tail moment and the length of DNA migration (tail length) were analyzed by Comet Assay IV software V4.3 (Perceptive Instruments, Suffolk, UK).

Animal study. All mouse experimental procedures were performed in accordance with the Regulations for the Administration of Affairs Concerning Experimental Animals approved by the State Council of the People's Republic of China. In addition, the present study was approved by the Ethics Committee of Shandong Provincial Hospital. All methods used in the present study were carried out in accordance with the approved guidelines.

BALB/c-nu mice (female, 5-6 weeks old) were purchased from the Vital River Laboratory Animal Technology (VRL; Beijing, China). Mice were bred and maintained under specific pathogen-free conditions. All animal experimental protocols were approved by the Ethics Committee of Shandong University. H520 cells $\left(4 \times 10^{6}\right)$ were subcutaneously injected into mice. Tumor growth was monitored and evaluated according to volume (length $\mathrm{x}$ width $\mathrm{x}$ width/2) at the indicated time-points during a 28-day period after the tumor volume reached $100 \mathrm{~mm}^{3}$. To assess the effect of ARRB1 on cisplatin in vivo, mice were divided into groups with matched weight: i) the group treated with $2.5 \mathrm{mg} / \mathrm{kg}$ cisplatin (control group); ii) the group treated with $2.5 \mathrm{mg} / \mathrm{kg}$ cisplatin after in vivo transfection of ARRB1 (ARRB1+ group). Cisplatin ( $2.5 \mathrm{mg} / \mathrm{kg}$ ) dissolved in normal saline was intraperitoneally injected every third day in both groups. ARRB1 plasmid $(1.5 \mathrm{mg} / \mathrm{kg})$ was intratumorally injected $24-48 \mathrm{~h}$ before cisplatin treatment in the ARRB1+ group.

Statistical analysis. All statistical calculations were performed using SPSS 19.0 (SPSS, Inc., Chicago, IL, USA) and GraphPad Prism 6 (GraphPad Software, Inc., La Jolla, CA, USA). Each experiment was performed in triplicate, at least 3 independent times. In addition, data are expressed in the form of count or mean \pm standard deviation, and analyzed by t-test or $\chi^{2}$ test according to the data characteristics (continuous variables, t-test; categorical variables, $\chi^{2}$ test). For all calculations, P-values were all two-sided and $<0.05$ was considered to indicate a statistically significant result. 
A
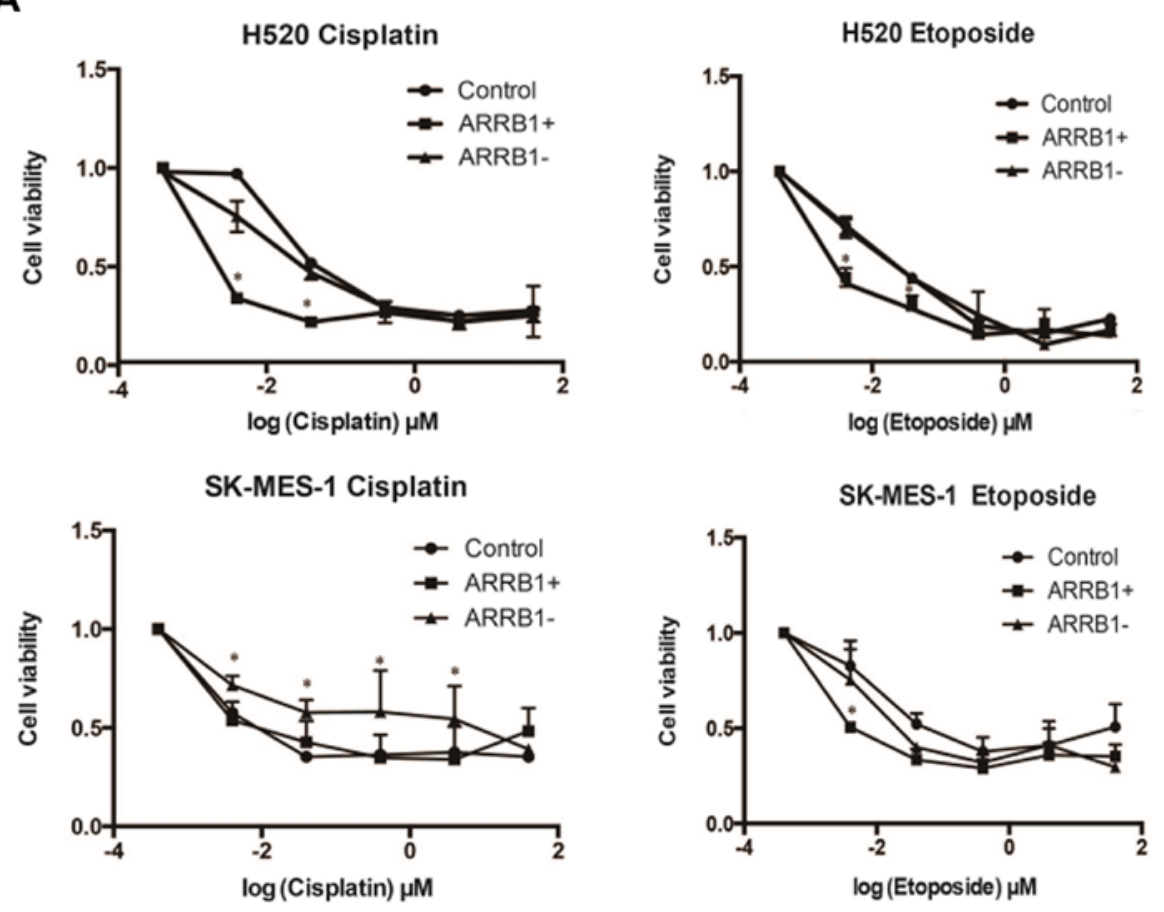

B

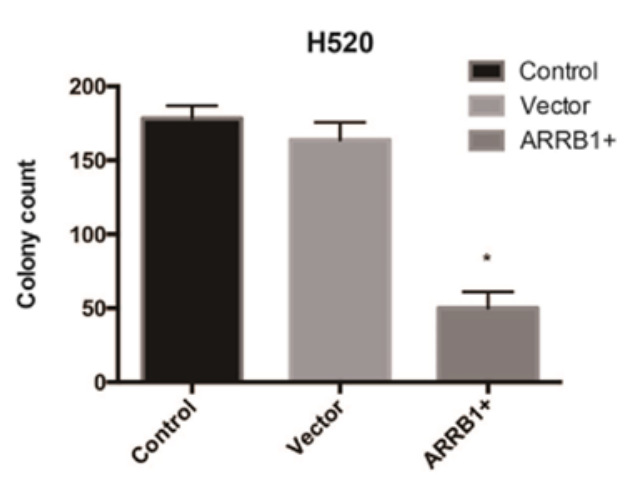

Figure 2. Effect of cisplatin and etoposide at various concentrations in H520 and SK-MES-1 cell lines with overexpression of ARRB1(+) or knockdown of ARRB1(-). The cell viability was evaluated by (A) MTT assay and (B) colony formation assay. ARRB1 overexpression enhanced the sensitivity of tumor cells to chemotherapy at lower concentrations and ARRB1-knockdown increased resistance to chemotherapy.

\section{Results}

Downregulation of ARRB1 in lung SCC patients. Characteristics of the patients are shown in Table I. Compared with para-carcinoma tissues, a significant downregulation of ARRB1 in SCC tissues was noted. Of the 30 matched cancer and normal tissues, the mRNA level of ARRB1 in 16 cancer tissues was decreased in comparison with this level in the matched para-carcinoma tissues. SCC patients with low levels of ARRB1 showed significantly poorer overall survival (OS) $(\mathrm{P}=0.0306)$ when compared with the OS in the patients with high ARRB1 (Fig. 1).

ARRB1 increases the efficacy of DNA-damaging agents in SCC in vitro. Cisplatin and etoposide were used in H520 and SK-MES-1 cell lines, respectively, at various concentrations and the resulting cell viability was assessed using MTT and colony formation assays (Fig. 2). The data we obtained suggested that ARRB1 enhanced the sensitivity of tumor cells to chemotherapy at lower concentrations and that ARRB1-knockdown increased resistance to chemotherapy.

ARRBI modulates the DDR pathway activated by $D N A$-damaging agents. We found that phosphorylated ATR and Chk1 were downregulated after ARRB1 knockdown. On the contrary, ATR and Chk1 maintained phosphorylation activation with ARRB1 transfection, which was in accordance with the status of BRCA1 activation (Fig. 3A and B). Downregulation of phosphorylated BRCA1, ATR and Chk1 indicated that DDR was inhibited. However, concurrently, downregulation of phosphorylated BRCA1 also indicated initiation of DNA repair. We also found that depletion of ARRB1 inhibited the phosphorylation of $\mathrm{H} 2 \mathrm{AX}$, which is a biomarker of DSBs. On the contrary, overexpression of ARRB1 increased the phosphorylation of $\mathrm{H} 2 \mathrm{AX}$, which was in accordance with the status of NF-kB phosphorylation (Fig. 3C and D). 
A

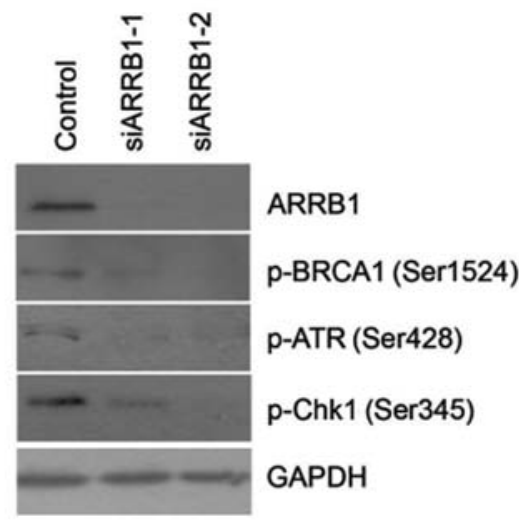

B

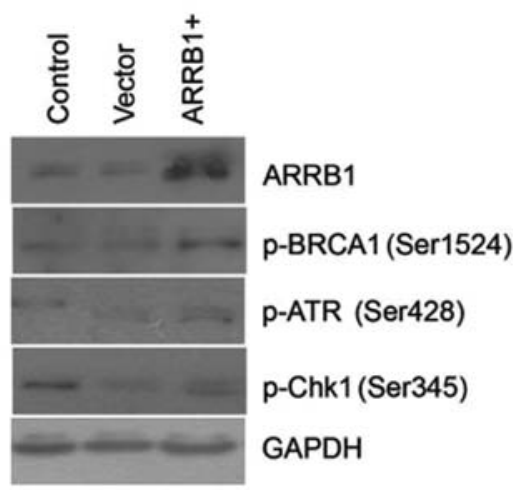

C

$\begin{array}{ccccccccc}\text { Cisplatin }(\mu M) & 0 & 0.1 & 1 & 10 & 0 & 0.1 & 1 & 10 \\ \text { ARRB1+ } & - & - & - & - & + & + & + & +\end{array}$

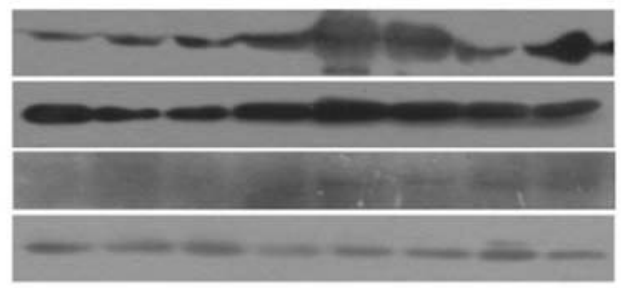

ARRB1

$Y-H 2 A X($ Ser139)

p-NF-kB (Ser536)

GAPDH

D

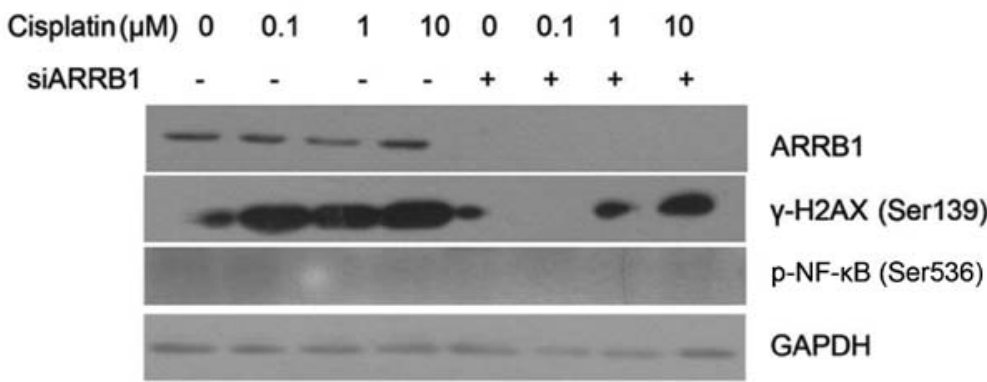

Figure 3. Expression of ARRB1, p-BRCA1, p-ATR, p-Chk1 and GAPDH in the H520 cell line (A) transfected with siARRB1-1 or siARRB1-2 or (B) the overexpression plasmid ARRB1(+) following treatment with $1.5 \mu \mathrm{M}$ cisplatin. The phosphorylated ATR and Chk1 were downregulated after ARRB1 knockdown; on the contrary, ATR and Chk1 maintained phosphorylation activation with ARRB1 transfection. Expression of ARRB1, $\gamma$-H2AX, p-NF- $\kappa$ B and GAPDH in the H520 cell line (C) transfected with the overexpression plasmid ARRB1(+) or (D) with siARRB1 following treatments with various concentrations of cisplatin. Depletion of ARRB1 inhibited the phosphorylation of H2AX, on the contrary, overexpression of ARRB1 increased the phosphorylation of H2AX.

ARRB1 enhances apoptosis and DNA damage induced by DNA-damaging agents. In order to quantitatively and precisely analyze the cellular responses and apoptosis, the FCM apoptosis and necrosis assay (Annexin V and 7-AAD) was performed in the experiments. In the present study, PE-Annexin V staining was used to detect the externalization of PS in apoptotic cells, and 7-AAD was applied to stain necrotic and dead cells, which lose their cell membrane integrity. Fig. $4 \mathrm{~A}$ and B, revealed that there were more apoptotic cells in the ARRB1-overexpressing cells compared with the control group, and less apoptotic cells in the ARRB1-knockdown cells. These are in accordance with the DNA damage results assessed by comet assay (Fig. 4C).

Overexpression of ARRBI leads to decreased tumor size in vivo. We examined the effect of ARRB1 overexpression via treatment with cisplatin on mouse xenograft models of lung cancer cells (H520). We observed a significant trend of decreased tumor growth with this treatment $(\mathrm{P}=0.038)$ (Fig. 5).

\section{Discussion}

In our research, we designed a series of experiments to explore the possible impacts of ARRB1 expression on chemotherapy efficacy in NSCLC and relevant mechanisms of regulation of ARRB1 in DDR induced by DNA-damaging agents. When cells detect DNA damage, DDR may immediately start. Then, the two key kinases ATR and ATM are activated. ATR and ATM are vital signaling molecules in the DDR pathway, which regulate the cell cycle, cell apoptosis and DNA repair (21). With the ATR-Chk1 and ATM-Chk2 pathways activated, Chk1 and Chk2 may be phosphorylated. Then phosphorylated-Chk1 (p-Chk1) phosphorylates the cdc25 family and other downstream substrates, which induce cell cycle arrest, DNA repair and other cellular responses. Similarly, phosphorylated-Chk2 (p-Chk2) phosphorylates p53 and the suppression of the combination of MDM2 and p53, causes p53 upregulation (22). Activated p53 enhances the expression of p21, 14-3-3o, 
A

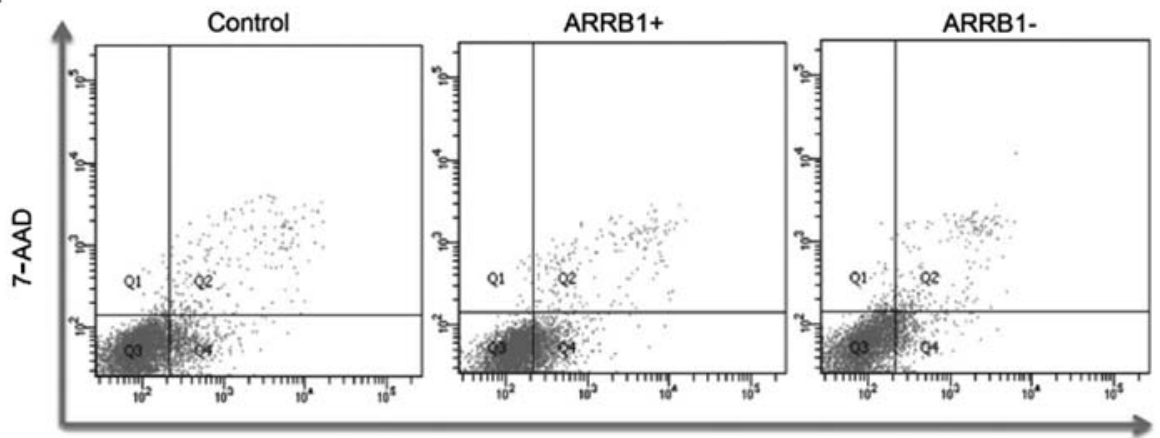

PE Annexin V

B

H520 Cisplatin

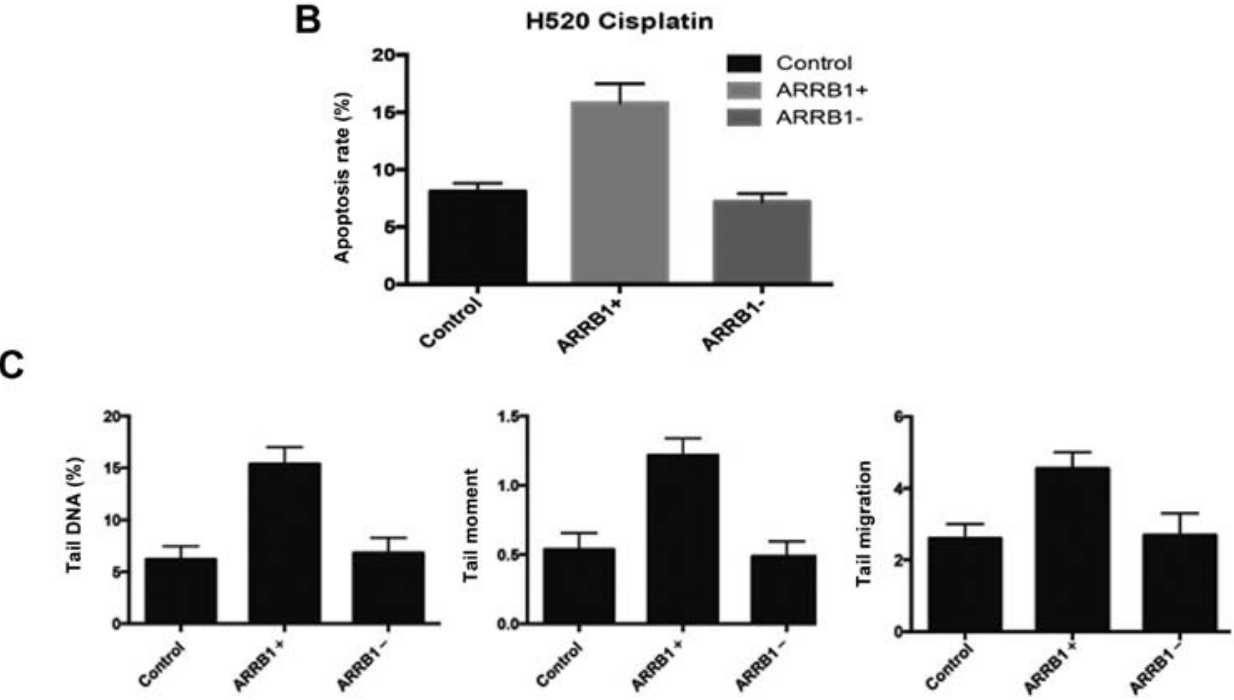

C

Figure 4. (A) Density plots drawn with Annexin V as the x-axis and 7-AAD as the y-axis. Necrotic cells are indicated at the upper left corner (Q1); dead or late apoptotic cells are indicated at the upper right corner (Q2); live cells are indicated at the lower left corner (Q3); early apoptotic cells are indicated at the lower right corner (Q4) in the plots. (B) A higher percentage of early apoptotic cells was noted in the ARRB-overexpressing cells compared with the control group, and a lower percentage of apoptotic cells was noted in the ARRB1-knockdown cells. (C) Three parameters (\% of tail DNA, tail length and tail moment) of the comet assay were all increased compared with the control group after transfection with ARRB1 plasmids.

A

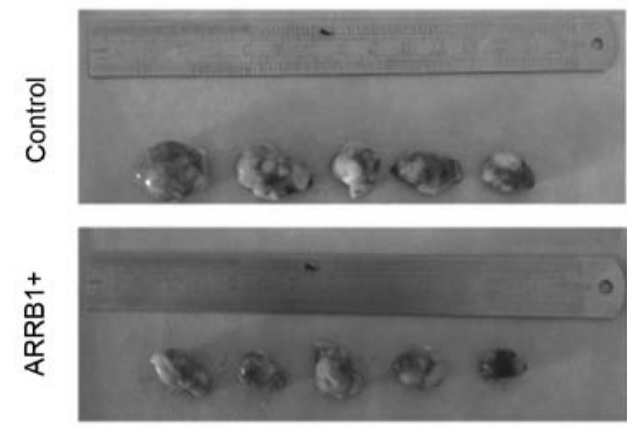

B

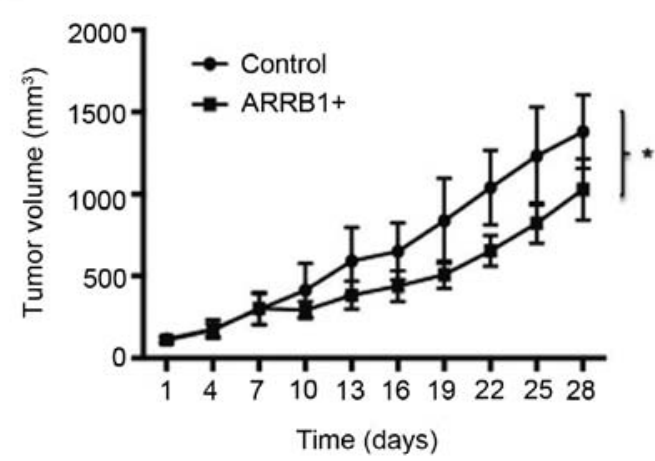

Figure 5. (A and B) ARRB1 transfection in vivo decreases tumor growth, when compared to the control group ( $\mathrm{P}=0.038)$. This indicated that tumors were more sensitive to cisplatin.

GADD45 and other signaling molecules, which ultimately causes cell cycle arrest. Thus, cells may have plenty of time to complete the DNA repair process. If the DNA repair process cannot be completed in time, it may lead to cell apoptosis (23).

Kook et al reported that ARRB1 is cleaved by caspases, and then binds to truncated BID (tBID) (24), which means that ARRB1 directly regulates apoptosis. Apart from that, our results revealed that more ATR and H2AX were phosphorylated compared with the control group after transfection with the ARRB1 plasmids. As a vital indicator of DNA DSBs, H2AX is required for checkpoint-mediated cell cycle arrest and DNA repair in DDR. That is to say, more 
DNA damage occurs after ARRB1 levels are increased. This fact also indicates that ARRB1 may enhance cell apoptosis and promote chemotherapy efficacy. The potential mechanism may be related with the interaction between ARRB1 and ATR and H2AX (25). ARRB1 is likely to be involved in the phosphorylation/activation of $\mathrm{H} 2 \mathrm{AX}$ and ATR. In contrast, low expression of ARRB1 can suppress cell proliferation through inhibition of cellular pathways such as the NF- $\kappa \mathrm{B}$ pathway. Activation of the $\mathrm{NF}-\kappa \mathrm{B}$ pathway was reported to be associated with chemoresistance and anti-apoptosis. Numerous studies have confirmed that ARRB1 in the nucleus promotes activation of the $\mathrm{NF}-\kappa \mathrm{B}$ pathway $(26,27)$. $\mathrm{NF}-\kappa \mathrm{B}$ is widely used by eukaryotic cells as a regulator of genes that control cell proliferation and cell survival. As such, many different types of human tumors have misregulated NF- $\kappa$ B. A previous study showed that co-expression of nuclear ARRB1 and $\mathrm{NF}-\kappa \mathrm{B}$ is associated with cancer progression and poor prognosis in lung adenocarcinoma (28), which indicates the potential relationship of ARRB1 and NF- $\kappa$ B.

In conclusion, ARRB1 participates in numerous cellular pathways and diversely functions under different circumstances. Our results and data showed that ARRB1 promoted DNA damage and cell apoptosis and depletion of ARRB1 inhibited DDR pathway activation. Thus, we surmise that ARRB1 may enhance chemosensitivity and be beneficial to cancer treatment. However, various clinical studies have reported that a high level of ARRB1 is related to a poor outcome in cancer treatment (29). Thus, the status of ARRB1 is associated with different tumor characteristics.

The roles of ARRB1 played in lung cancer treatment have not been extensively studied, and in particular its clinical relevance has not been previously addressed. The detailed and concrete functions of ARRB1 in lung cancer treatment require further study.

\section{Acknowledgements}

The present study was supported by the National Natural Science Foundation of China (project 81301728), the Key Research and Development Program of Shandong Province (2015GSF118129), the Foundation for Outstanding Young Scientist in Shandong Province (BS2013YY066), and the Medicine and Health Science Technology Development Plan of Shandong Province (2013WS0103).

\section{References}

1. Siegel RL, Miller KD and Jemal A: Cancer statistics, 2015. CA Cancer J Clin 65: 5-29, 2015

2. Ferlay J, Soerjomataram I, Dikshit R, Eser S, Mathers C, Rebelo M, Parkin DM, Forman D and Bray F: Cancer incidence and mortality worldwide: sources, methods and major patterns in GLOBOCAN 2012. Int J Cancer 136: E359-E386, 2015.

3. Jin F, Zhu H, Shi F, Kong L and Yu J: A retrospective analysis of safety and efficacy of weekly nab-paclitaxel as second-line chemotherapy in elderly patients with advanced squamous nonsmall-cell lung carcinoma. Clin Interv Aging 11: 167-173, 2016.

4. Tan HL, Ang YL and Soo RA: Therapeutic options in advanced squamous cell lung carcinoma. Lung Cancer 4: 75-86, 2015.
5. Koutsoukos K and Mountzios G: Novel therapies for advanced squamous cell carcinoma of the lung. Future Oncol 12: 659-667, 2016.

6. Cufer T and Knez L: Update on systemic therapy of advanced non-small-cell lung cancer. Expert Rev Anticancer Ther 14: 1189-1203, 2014.

7. Rothschild SI: Advanced and metastatic lung cancer - what is new in the diagnosis and therapy? Praxis 104: 745-750, 2015 (In German).

8. Hosoya $\mathrm{N}$ and Miyagawa K: Targeting DNA damage response in cancer therapy. Cancer Sci 105: 370-388, 2014.

9. Jackson SP and Bartek J: The DNA-damage response in human biology and disease. Nature 461: 1071-1078, 2009.

10. Ciccia A and Elledge SJ: The DNA damage response: Making it safe to play with knives. Mol Cell 40: 179-204, 2010.

11. Lefkowitz RJ and Whalen EJ: beta-arrestins: Traffic cops of cell signaling. Curr Opin Cell Biol 16: 162-168, 2004.

12. Lefkowitz RJ and Shenoy SK: Transduction of receptor signals by beta-arrestins. Science 308: 512-517, 2005.

13. Sobolesky PM and Moussa O: The role of $\beta$-arrestins in cancer. Prog Mol Biol Transl Sci 118: 395-411, 2013.

14. Ma H, Wang L, Zhang T, Shen H and Du J: Loss of $\beta$-arrestin1 expression predicts unfavorable prognosis for non-small cell lung cancer patients. Tumour Biol 37: 1341-1347, 2016.

15. Hara MR, Kovacs JJ, Whalen EJ, Rajagopal S, Strachan RT, Grant W, Towers AJ, Williams B, Lam CM, Xiao K, et al: A stress response pathway regulates DNA damage through $\beta_{2}$-adrenoreceptors and $\beta$-arrestin-1. Nature 477: 349-353, 2011.

16. Hara MR, Sachs BD, Caron MG and Lefkowitz RJ: Pharmacological blockade of a $\beta_{2}$ AR- $\beta$-arrestin- 1 signaling cascade prevents the accumulation of DNA damage in a behavioral stress model. Cell Cycle 12: 219-224, 2013.

17. van Engeland M, Nieland LJ, Ramaekers FC, Schutte B and Reutelingsperger CP: Annexin V-affinity assay: A review on an apoptosis detection system based on phosphatidylserine exposure. Cytometry 31: 1-9, 1998.

18. Zembruski NC, Stache V, Haefeli WE and Weiss J: 7-Aminoactinomycin D for apoptosis staining in flow cytometry. Anal Biochem 429: 79-81, 2012.

19. Patra B, Peng CC, Liao WH, Lee CH and Tung YC: Drug testing and flow cytometry analysis on a large number of uniform sized tumor spheroids using a microfluidic device. Sci Rep 6: 21061, 2016.

20. Fairbairn DW, Olive PL and O'Neill KL: The comet assay: A comprehensive review. Mutat Res 339: 37-59, 1995.

21. Maréchal A and Zou L: DNA damage sensing by the ATM and ATR kinases. Cold Spring Harb Perspect Biol 5: a012716, 2013.

22. Cheng Q and Chen J: Mechanism of p53 stabilization by ATM after DNA damage. Cell Cycle 9: 472-478, 2010.

23. Zhou BB and Elledge SJ: The DNA damage response: Putting checkpoints in perspective. Nature 408: 433-439, 2000.

24. Kook S, Zhan X, Cleghorn WM, Benovic JL, Gurevich VV and Gurevich EV: Caspase-cleaved arrestin-2 and BID cooperatively facilitate cytochrome $c$ release and cell death. Cell Death Differ 21: 172-184, 2014.

25. Xiao K, McClatchy DB, Shukla AK, Zhao Y, Chen M, Shenoy SK, Yates JR III and Lefkowitz RJ: Functional specialization of $\beta$-arrestin interactions revealed by proteomic analysis. Proc Natl Acad Sci USA 104: 12011-12016, 2007.

26. Bernal-Mizrachi L, Lovly CM and Ratner L: The role of NF-kB-1 and NF- $\kappa \mathrm{B}-2$-mediated resistance to apoptosis in lymphomas. Proc Natl Acad Sci USA 103: 9220-9225, 2006.

27. Cianfrocca R, Tocci P, Semprucci E, Spinella F, Di Castro V, Bagnato $A$ and Rosanò L: $\beta$-Arrestin 1 is required for endothelin-1-induced NF- $\kappa$ B activation in ovarian cancer cells. Life Sci 118: 179-184, 2014.

28. Yu J, Wang L, Zhang T, Shen H, Dong W, Ni Y and Du J: Co-expression of $\beta$-arrestin 1 and $N F-\mathrm{KB}$ is associated with cancer progression and poor prognosis in lung adenocarcinoma. Tumour Biol 36: 6551-6558, 2015.

29. Lundgren K, Tobin NP, Lehn S, Stål O, Rydén L, Jirström K and Landberg G: Stromal expression of $\beta$-arrestin- 1 predicts clinical outcome and tamoxifen response in breast cancer. J Mol Diagn 13: 340-351, 2011. 